\title{
LA ONTOLOGÍA TRAS BASTIDORES. UN PROBLEMA METAFÍSICO PARA EL NATURALISMO GENÉTICO
}

\author{
Miguel Ángel Pérez Jiménez* \\ doi:10.11144/Javeriana.uph33-66.obpm
}

\begin{abstract}
RESUMEN
Este trabajo es una crítica de ciertas concepciones de lo que llamamos una perspectiva genética de la naturalización: la tarea de investigar cómo la razón emerge del mundo natural. Para desarrollar nuestra crítica tomamos como marco de referencia dos importantes propuestas de Donald Davidson: el monismo anómalo y la analogía de la triangulación. Nuestro problema es el carácter metafísico del monismo y sus implicaciones para la empresa genética de naturalización de la razón. Defendemos que la concepción metafísica de la naturaleza del monismo anómalo, subyacente en la analogía de la triangulación, impide que la pregunta genética pueda plantearse de un modo inteligible y que, en esa medida, pueda aspirar a una respuesta razonable.
\end{abstract}

Palabras clave: monismo anómalo; triangulación; naturalismo; Donald Davidson

* Pontificia Universidad Javeriana, Bogotá, Colombia.

Correo electrónico: miguel.perez@javeriana.edu.co

Para citar este artículo: PÉREZ JimÉNEZ, M.A. (2016). La ontología tras bastidores. Un problema metafísico para el naturalismo genético. Universitas Philosophica, 33(66), pp. 61-76.

ISSN 0120-5323, ISSN en línea: 2346-2426, doi:10.11144/Javeriana.uph33-66.obpm

Este trabajo, que comenzó durante una estancia de investigación en el Departamento de Filosofía I de la Universidad de Granada, España, y que contó con el apoyo financiero de LASPAU, Colciencias y la Pontificia Universidad Javeriana de Bogotá, Colombia, es resultado parcial del proyecto de investigación: Triangulación y atención conjunta. Revisión crítica del debate y una propuesta experiencial expresivista, ID PRY 006111 de la Vicerrectoría de Investigación de la Pontificia Universidad Javeriana de Bogotá. Agradezco a Juan José Acero sus valiosos comentarios a una versión previa del material. Agradezco también a Camila Suárez y a Andrés Pinilla sus aportes en la construcción del mismo. 


\title{
BACKSTAGE ONTOLOGY. A CRITICISM OF GENETIC NATURALISM
}

\author{
Miguel Ángel Pérez Jiménez
}

\begin{abstract}
This paper criticizes genetic naturalism, namely, the task of explaining how reason emerges from nature. We take into account Donald Davidson's anomalous monism and his analogy of triangulation. Our aim is to criticize both the metaphysical suppositions of anomalous monism and its consequences for genetic naturalism. We propose that those suppositions and those consequences prevent the genetic question to be appropriately raised and, therefore, mislead the research for a reasonable answer to that.

Key words: anomalous monism; triangulation; naturalism; Donald Davidson
\end{abstract}


ESTE TRABAJO TIENE UN OBJETIVO crítico y se dirige a ciertas concepciones de lo que llamaremos una perspectiva genética de la naturalización: la tarea de investigar cómo la razón emerge del mundo natural. Para desarrollar nuestra crítica tomaremos como marco de referencia el monismo anómalo y la analogía de la triangulación. Aunque estas teorías han sido repetidamente criticadas, en especial por las anomalías de lo mental ${ }^{1}$, nuestro problema es el carácter metafísico del monismo y sus implicaciones para la empresa genética de naturalización de la razón. La tesis que defendemos es que la concepción metafísica de la naturaleza del monismo anómalo impide que la pregunta genética pueda plantearse de un modo inteligible y que, en esa medida, pueda aspirar a una respuesta razonable.

\section{Las triangulaciones y la emergencia de la razón}

El MONISMO ANÓMALO ES UNA TEORÍA acerca de la naturaleza causal de los fenómenos mentales y del irreducible carácter racional de la explicación psicológica (Davidson, 1970); sin embargo, no afirma cosa alguna acerca de cómo la naturaleza engendra la razón. El monismo anómalo no es una teoría genética. Para esta teoría la explicación psicológica se enmarca en una teoría interpretativa de la acción. Cuando el comportamiento de una criatura es suficientemente complejo como para no poder ser explicado causalmente, empleamos atribuciones de vocabulario psicológico para hacerlo (Davidson, 1997, p. 185). Dichas atribuciones se rigen por dos principios de caridad. En primer lugar, por el principio de coherencia, que pide considerar el comportamiento del agente conforme a un conjunto amplio y consistente de creencias, y de otras actitudes proposicionales. En segundo lugar, por el principio de correspondencia, según el cual las actitudes que se atribuyen a un agente deben ser acertadas en general respecto al mundo según el criterio del propio intérprete (Davidson, 1991, p. 288). Este ejercicio interpretativo por el que la explicación de la acción está sujeta a una atribución de actitudes proposicionales constreñida por los principios constitutivos de la racionalidad se conoce como racionalización (Davidson, 1963, p. 17; 1970, p. 361).

\footnotetext{
1 Otro tanto cabe decir sobre las críticas acerca de las dificultades de la teoría para explicar la causa-
} lidad mental y la acción intencional. Al respecto, véase: Moya, 1990, p. 112ss; Kim, 1998, p. 138s. 
La impronta de la racionalidad según esta concepción es su carácter proposicional (Davidson, 1982, p. 142). Con él se alude al menos a cuatro características de la razón: es holista en sentido conceptual, exhibe consistencia relativa entre creencias, involucra relaciones entre creencias y actitudes evaluativas, y es causa de las acciones ${ }^{2}$. Esta caracterización de la racionalidad otorga un lugar prominente a las actitudes proposicionales cuya característica definitoria, desde un punto de vista semántico, es su intensionalidad. A diferencia de Quine, Davidson no rechazó este tipo de actitudes porque las consideraba constitutivas de la interpretación de las acciones racionales, de modo que la intensionalidad que las caracteriza pasó a ser un aspecto decisivo de su teoría sobre el lugar de la razón en la naturaleza.

Por otra parte, en el monismo anómalo la teoría interpretativa de la racionalidad se considera compatible con una concepción causal de la naturaleza de los estados psicológicos ${ }^{3}$, lo que permite mantener un monismo ontológico. Tras una concepción singular de la causalidad, se afirma que los estados mentales son eventos causales en tanto admiten una descripción verdadera según la cual son instancias de una ley de la naturaleza ${ }^{4}$. Si dicha descripción, en conjunción con otros enunciados, es una instancia de una ley estricta, entonces la explicación será de carácter nomológico. El monismo no es el resultado de una observación acerca del funcionamiento de la explicación causal, sino un postulado metafísico. Las explicaciones nomológicas del evento causal son intensionales, mientras que las relaciones causales mismas son extensionales (Macdonald, 1989, p. 85)

El monismo anómalo no es una teoría genética y no puede serlo, ya que como explicación de la aparición de la razón en la naturaleza resultaría circular, dada su presuposición de la racionalización. La analogía de la triangulación, por el contra-

2 Sobre estas características, véase: Davidson, 1997, p. 177ss; Lepore et al., 2005, p. 212s; Malpas, 1992, p. 86ss.

3 Davidson defendió a su vez dos tipos de anomalía de lo mental: no hay leyes psicofísicas estrictas ni tampoco leyes psicológicas estrictas (Davidson, 1970, p. 273).

4 Véase: Davidson, 1963, p. 33s; Macdonald, 1989, p. 84s; Evnine, 1991, p. 35.

5 Por eso algunos como Glock (2003, p. 33) Heal (1997, p. 176) y Ramberg (1989, p. 71) han llegado a decir que el proyecto filosófico de este autor consiste en mostrar cómo lo intensional se deriva de lo extensional. 


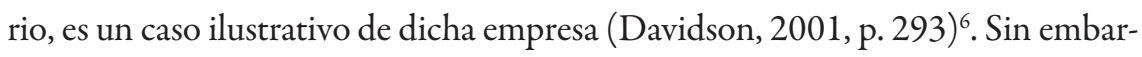
go, la explicación genética triangular no pretende ser una explicación evolutiva acerca de cómo la naturaleza engendra la razón ${ }^{7}$, sino, más bien, un intento de dar las condiciones necesarias y suficientes de la racionalidad. También, en consonancia con el monismo anómalo, la triangulación procura respetar la especificidad del vocabulario psicológico y conservar una unidad ontológica natural. Precisamente por ello, como veremos, reaviva una vieja y polémica inquietud: cómo reconciliar nuestra comprensión causal y normativa de los estados mentales.

Las triangulaciones rompen la circularidad dibujada en el monismo anómalo por la teoría interpretativa de la acción y los principios de caridad, introduciendo un escenario explicativo que prescinde por completo de aspectos racionales y que conserva todas las características causales de las situaciones interpretativas radicales. "La situación básica es una que involucra dos o más criaturas simultáneamente en interacción unas con otras y con el mundo que comparten; es lo que llamo triangulación" (Davidson, 1997, p. 182). Esta situación se da tanto entre criaturas racionales como entre criaturas que interactúan solo causalmente, como un banco de peces; razón por la cual puede catalogarse como una condición natural de la atribución de racionalidad. Por eso se considera que sin otros seres con los cuales interactuar y sin un entorno que sirva de escenario y referente de la interacción no es posible que emerja la racionalidad en la naturaleza.

Esta triangulación causal, no obstante, es solo una condición necesaria de la aparición de la razón. Para completar la tarea se introduce un segundo tipo de triangulación como condición suficiente, a saber: la nueva triangulación es la interacción comunicativa entre las criaturas conceptuales, caracterizada al modo de las situaciones clásicas de interpretación radical ${ }^{8}$. Como consecuencia solo podemos atribuirle estados de actitud proposicional a una criatura cuando puede hablar. El comportamiento lingüístico es el único suficientemente complejo

6 La exposición más clara de la aplicación que aquí consideramos de la triangulación es Davidson, 1997. El principal interés en ese texto es el problema conceptual de cómo atribuir vocabulario psicológico con base en evidencias completamente naturales. Nuestro interés presente es el problema metafísico. El problema conceptual lo hemos elaborado en Pérez, 2008.

7 Sobre el pesimismo de Davidson al respecto y algunas alternativas a su posición puede verse: Davidson, 1997, p. 182; Pérez, 2008.

8 Acerca de esta triangulación, véase: Davidson, 1997, p. 185 y Glüer, 2006, p. 1012. 
como para motivar la atribución de actitudes proposicionales a un agente, tesis que, por supuesto, conlleva la imposibilidad de atribuir racionalidad a los animales y a los niños muy pequeños.

Como el monismo anómalo, la explicación de las condiciones de la racionalidad por vía de las triangulaciones es insuficiente desde un punto de vista genético, pues simplemente enuncia una condición extensional necesaria para lo intensional, una condición natural de la racionalidad, pero sigue dejando en el vacío cómo de la naturaleza emerge la razón?. La dificultad reposa en que las triangulaciones se han definido como compartimentos completamente diferentes y sin ninguna relación entre sí, de modo que todo contacto entre lo extensional y lo intensional ha quedado por fuera, cuando solo la exploración de ese encuentro puede responder la cuestión genética ${ }^{10}$.

Algunos autores han creído que el problema de la triangulación para explicar genéticamente en sentido evolutivo se debe a la incompatibilidad misma que tienen los conceptos de causalidad y de normatividad. El propio Davidson quizá haya sido el más perplejo con la dificultad al notar que carecíamos de un vocabulario intermedio entre el causal y el racional, y que por tanto toda explicación evolutiva estaba condenada desde el principio (Davidson, 1997, p. 182). Pero no ha sido el único. Otros también se han quejado por el vacío abierto entre el reino de lo causal y el de lo racional, como es el caso de Eilan (2005, p. 5) y Miguens (2006, p. 104), e incluso hay quienes lo han tomado como razón suficiente para condenar la empresa davidsoniana por completo, como Glock (2003, pp. 11, 262s) y Miguens (2006, p. 116) ${ }^{11}$.

9 Véase: Eilan, 2005, p. 12; Glüer, 2006, p. 1013; Miguens, 2006, p. 104.

10 En sentido estricto, esta crítica acarrea la afirmación de que Davidson no logra llevar a buen término su proyecto filosófico, pues jamás muestra cómo lo intensional se deriva de lo extensional. En todo caso, el propio Davidson ha argumentado que no es posible dar condiciones necesarias y suficientes de la racionalidad en términos naturalistas, pues ello supondría la posibilidad de reducción del vocabulario intensional al extensional, algo que por los principios constitutivos de la racionalidad está excluido de entrada. Sobre este punto, ver: Davidson, 2001, p. 293; Glüer, 2006, p. 1013.

11 Estas réplicas son el eco de unas más clásicas que atacaban ya el monismo anómalo poniendo énfasis en la incompatibilidad entre la concepción a la vez causal y normativa de los estados mentales. Davidson enfrentó las principales críticas en el texto de 1963, se trata del famoso argumento de la conexión lógica, como se puede rastrear en Moya (1990, pp. 106, 110). Dichas objeciones han motivado al menos cuatro tipos diferentes de alternativas. Confiados en la corrección de las 
En lo que sigue intentaremos desarrollar un argumento crítico alternativo con el que pretendemos defender a Davidson y a sus críticos, y además reivindicar un sentido que todavía otorga plausibilidad a la empresa genética. Para hacerlo son necesarias unas observaciones metafísicas preliminares.

2. Dos tipos de criaturas triangulantes y la normatividad de la verdad

CON LA EXPLICACIÓN GENÉTICA por vía de las triangulaciones no solo asumimos la circularidad metodológica del monismo anómalo, introducida por la teoría interpretativa de la acción y por el principio de caridad, sino también una circularidad metafísica. Esta circularidad funciona a dos niveles: respecto a la naturaleza de las criaturas que triangulan y, de un modo diferente, respecto al entorno que sirve como tercer elemento de la triangulación.

La diferencia metafísica entre los seres que satisfacen solo la condición extensional y los que satisfacen también la intensional, es que los primeros pueden darse cuenta de lo que pasa en el entorno y de lo que hacen otras criaturas gracias a sus capacidades de reconocimiento y discriminación, mientras que los segundos lo hacen mediante sus capacidades de clasificación (Davidson, 1991, p. 285; 1997, p. 177). La diferencia entre estos dos tipos de capacidades es que las primeras solo permiten establecer diferencias en ciertos rasgos del entorno, mientras que las segundas permiten un grado fino de representación: la generalidad. En pocas palabras, los seres que solo discriminan, como los ratones, no tienen conceptos, los que pueden clasificar sí los tienen.

Decir que una criatura clasifica es otorgarle una dimensión normativa a sus capacidades perceptivas (Davidson, 1997, p. 177). Para que una clasificación sea normativa es preciso que podamos equivocarnos en su ejecución y, puesto que los conceptos se ejecutan en el juicio, entonces el fallo en su aplicación debe buscarse

críticas algunos autores han instado a Davidson a abandonar su compromiso con la causalidad (McDowell, 1985), mientras que otros han insistido en su explicación naturalizada de la racionalidad (Fodor, 1994). Por otra parte, hay quienes han motivado una orientación hacia fenómenos más básicos, como la intencionalidad, para explorar un terreno en el que las dicotomías no aparezcan (Moya, 1992), y quienes han buscado una perspectiva de segunda persona que evite las unilateralidades a las que conducen la perspectiva causal de la tercera persona y racional de la primera persona (Miguens, 2006, p. 113; Pinedo, 2004, p. 228; Pérez, 2008, p. 188ss). 
en él. El fallo en el juicio es su falsedad, de modo que la aplicación errónea del concepto se deriva de la falsedad del juicio en que aparece. Por esta razón podemos decir que la verdad es la normatividad de lo conceptual, pero no solo eso.

Un juicio falso supone un error de clasificación pero no de racionalidad. Por el contrario, cuando una criatura no logra ni siquiera construir un juicio no puede atribuírsele racionalidad y, entonces, en este caso se la debe explicar causalmente. De este modo, la normatividad de la verdad no es solo la impronta de lo conceptual y la marca de lo intensional sino, también, un criterio para trazar la distinción metafísica entre las criaturas racionales y las simplemente naturales.

\section{Un argumento semántico a favor del monismo}

LAS TRIANGULACIONES SE HAN DEFINIDO de modo excluyente. La primera es causal, la segunda, normativa; la primera es no conceptual, la segunda, sí; la primera es extensional, la segunda, intensional. Estas diferencias establecen distinciones metodológicas a nivel de las explicaciones (explicación causal/ racionalización), conceptuales a nivel de las condiciones de uso de los conceptos causales y psicológicos, y también a nivel metafísico. En este último campo han tomado la forma de la distinción entre criaturas no conceptuales y criaturas conceptuales. Sin embargo, no parecen impactar en la concepción del entorno: el monismo del monismo anómalo parece todavía intacto por las tenaces fragmentaciones de Davidson. A continuación presentaremos un argumento semántico para defender este monismo.

En el caso de la triangulación conceptual empleamos vocabulario intensional para describir las interacciones. La característica de este es que al aplicarlo las expresiones que construimos no pasan la prueba de sustitución salva veritate (Davidson, 1975, p. 171s). En el caso de la triangulación preconceptual empleamos vocabulario extensional, y con ello admitimos que su descripción puede hacerse en expresiones preservadoras de la verdad según la misma prueba de sustitución. Así, desde un punto de vista semántico, resulta que el rasgo común a las dos triangulaciones es que el tipo de interacciones que hay en ellas depende positiva o negativamente de la prueba de sustitución de expresiones correferenciales. Cuando se pasa la prueba, estamos en triangulaciones preconceptuales; cuando no, estamos en triangulaciones conceptuales. 
Dicho esto, podemos reconocer que la intensión está construida sobre el molde de la extensión, pero de un modo opuesto: intensional es aquello que no pasa las pruebas de extensionalidad ${ }^{12}$. Solo disponemos de una definición negativa de la intensionalidad y, en consecuencia, hay una caracterización recíproca y excluyente de la intensionalidad y de la extensionalidad, representada en la prueba de sustitución. Causalidad extensional y racionalidad intensional son excluyentes. ¡Por eso los detractores de Davidson tienen razón! No se pueden derivar una de la otra.

La pregunta es, entonces, ¿qué expresiones admiten que se les aplique la prueba? La respuesta es: las proposiciones. Una proposición que se ajuste a la ley de Leibniz es la representación de un hecho en términos de expresiones individuales y predicados. Precisamente, la prueba se define por la posibilidad que hay de combinar los predicados con diversas expresiones individuales y viceversa. Pero si esto es así, llama la atención que esta exigencia supone un mundo de hechos constituidos por objetos y propiedades. Esto se debe, como dijimos, a la concepción davidsoniana de la causalidad que, aunada a una definición de la verdad en términos de satisfacción, conduce a pensar que para que un evento sea causal debe poder ser descrito en términos proposicionales, es decir, en términos de predicados, individuos y proposiciones. Puesto que esto se acepta sin más, entonces al definir recíprocamente la intensión y la extensión mediante la prueba de sustitución, es posible admitir un mismo mundo natural causal para las dos triangulaciones: el que se representa proposicionalmente. ¡Por eso Davidson tiene razón!

Que tanto Davidson como sus opositores tengan razón nos pone en la penosa situación de afirmar que la empresa genética no es plausible. Si aceptamos que naturaleza y razón son conceptos excluyentes, carece de sentido preguntar cómo

12 Así desde Carnap y Quine. "Se dice que una oración es extensional con respecto a un designador que contiene si la extensión de la oración es una función de la extensión del designador; es decir, si el reemplazo de un designador por uno equivalente transforma la oración completa en una equivalente. Se dice que un oración es intensional con respecto a un designador que contiene si no es extensional y si su intensión es una función de la intensión del designador; es decir, si el reemplazo de este designador por uno L-equivalente transforma la oración completa en una L-equivalente" (Carnap, 1956, p. 1). "Un contexto es extensional si su valor de verdad no puede ser modificado mediante la sustitución de una oración componente por otra del mismo valor de verdad, ni con la sustitución de un predicado componente por otro con los mismos denotata, ni con la sustitución de un término singular por otro con el mismo designatum. Por decirlo con mayor brevedad, los tres requerimientos son: sustituibilidad de covalentes, sustituibilidad de coextensionales y sustituibilidad de idénticos, salva veritate. Un contexto es intensional si no es extensional" (Quine, 1998, p. 104). 
se deriva una de la otra. Por otra parte, si queremos preservar el valor del vocabulario psicológico no podemos acoger el eliminitavismo, con lo que la única alternativa que queda es el conceptualismo, pero allí estamos simplemente presos de un encierro conceptual que nada aporta a la pregunta genética ${ }^{13}$.

\section{Una concepción inconsistente de la naturaleza}

PARA ENFRENTAR CRÍTICAMENTE ESTA TESIS de un mundo natural causal de hechos, objetos y propiedades, pondremos en contacto las dos triangulaciones de Davidson. No buscamos ya un caso de interacción entre criaturas preconceptuales o entre criaturas conceptuales, sino uno de interacción entre una criatura preconceptual y una conceptual que llamaremos triangulación transicional. En este nuevo escenario decimos que las dos criaturas interactúan entre sí y respecto a una realidad independiente de ellas. Si admitimos que esa realidad independiente está estructurada ya en objetos, propiedades y hechos, estamos afirmando que tanto para la criatura preconceptual como para la conceptual la realidad tiene ya esa forma. El argumento semántico esbozado parece conducirnos a la aceptabilidad de esta conclusión. Sin embargo, intuitivamente parece poco plausible. ¿Acaso para los peces el mundo se aparece bajo la forma de objetos y propiedades?, ¿son hechos definidos proposicionalmente eso con lo que el bebé interactúa? ?4 $^{14}$

Empezaremos con la siguiente pregunta: ¿qué condiciones debe satisfacer una criatura para reconocer un objeto como objeto y una propiedad como propiedad? Una respuesta a esta cuestión es la que ofreció Gareth Evans (1982, p. 100ss) con la "restricción de generalidad". Si una criatura sabe que algo es un objeto con una determinada propiedad, entonces debe poder asociar ese objeto a otras propiedades, y a esa propiedad otros distintos objetos. La determinación de qué cuenta como objeto o qué como una propiedad es una habilidad que solo se puede tener si se tiene concepto de otras propiedades o de otros objetos respectivamente ${ }^{15}$.

13 Desarrollamos el argumento en Pérez, 2008.

14 El problema ya fue notado por Hornsby (1985) y Evnine (1991, p. 64s).

$15 \mathrm{Al}$ respecto véanse, también, las posturas de Günther (2003, p. 9) y Tye (2006, p. 9). 
El teórico de la interpretación radical podría no tener problemas para aceptar la restricción de generalidad, pues, después de todo, ella se acompasa bien con la prueba de sustitución (Davidson, 1982, p. 145). Lo que resulta curioso es que si pensamos en las implicaciones de aceptar dicha restricción para la triangulación transicional encontramos problemas como el siguiente. Si el adulto y el bebé están en el mismo mundo especificado en términos de objetos y propiedades, entonces resulta que si, por ejemplo, el adulto piensa en su gato, 'felix', jugando, entonces el bebé estará también en una relación con el gato juguetón. El adulto podrá creer que “el gato está jugando", pero el niño no podrá creer eso, no podrá tener la actitud proposicional de creer que el gato juega. Sin embargo, el bebé estará en una relación causal con el gato que juega. Si esto es así, la prueba de sustitución nos priva de atribuirle actitudes proposicionales al bebé, pero no de atribuirle contenidos ${ }^{16}$. Esos contenidos son los que presentan un mundo estructurado en términos de objetos y propiedades, tanto para lo seres con actitudes como para los que no las tienen.

No obstante, podemos preguntarnos si las criaturas conceptuales y las no conceptuales solo difieren en las actitudes y no en los contenidos. Esto es en todo caso lo que afirma el monismo anómalo. Según la restricción de generalidad, se tiene el contenido 'gato' en virtud de la posesión de otros contenidos con los que se compone bien ${ }^{17}$ : 'es animal', 'es felino', etc. Así, para que el bebé tuviera el contenido de "el gato juega" tendría que poder tener al menos algunos otros contenidos como "el gato es animal" o "el animal juega”. ¿Podemos atribuir también estos contenidos al bebé? De no poder hacerlo tampoco podríamos atribuir el primero.

Los teóricos del contenido no conceptual han reconocido que hay una diferencia importante respecto a las propiedades de los individuos a los que se les atribuyen contenidos. Muchas veces es posible atribuir contenidos a una criatura sin que ello implique que tenga esos conceptos, pero también hay casos en los que la atribución de contenidos supone que la criatura tenga esos conceptos ${ }^{18}$.

16 Es polémico qué pueda afirmarse de las criaturas que no pasan la prueba de sustitución. Davidson (1982, p. 148s) parece admitir que a una criatura así a lo mejor no puede atribuírsele mucho pensamiento, pero sí algo de pensamiento.

17 La restricción de generalidad de Evans supone algunas otras restricciones adicionales, pues es claro que las combinaciones posibles de nombres y predicados exceden las que la composicionalidad semántica admitiría.

18 Véase: Günther (2003, p. 9) y Tye (2006, p. 10). 
En este orden de ideas se han definido las propiedades conceptuales como aquellas que "se caracterizan canónicamente en relación con una teoría por medio de conceptos tales que el organismo necesita tener para satisfacer la propiedad" (Cussins, 1990, p. 382).

Según esta definición, si el bebé tiene el contenido 'gato', puesto que ese contenido solo puede especificarse por relación con otros contenidos como 'ser animal' o 'ser felino', entonces tenemos que decir que el bebé debe tener esos conceptos. Si esto es así, al atribuirle el primer contenido suponemos que el bebé tiene, por tanto, propiedades conceptuales. Esto resulta problemático desde un punto de vista metafísico, pues nos muestra un tipo de criaturas con propiedades conceptuales que pueden reconocerse incluso antes de aplicar la prueba de sustitución que, como vimos, termina siendo el baremo con el que se trazan estas diferencias ontológicas en la interpretación radical.

Se podría objetar que tendenciosamente hemos escogido una descripción del mundo en términos de 'gato' y 'juego' en lugar de 'cambiantes manchas deformes' y que por eso hemos llegado a la paradójica conclusión de que las criaturas no conceptuales tienen propiedades conceptuales. Esta objeción obliga a reconsiderar que la descripción del contenido que se emplee en estos casos debe ser una descripción canónica del contenido. Al respecto, cabe recordar que "ciertas especificaciones de un estado o de una actividad se identifican dentro de una teoría del contenido como siendo canónicas cuando son especificaciones generadas por la teoría en orden a capturar la manera distintiva en que algún aspecto del mundo se da al sujeto del estado o de la actividad" (Cussins, 1990, p. 384).

La descripción canónica del contenido debe darse conforme a una teoría que captura la manera de darse el mundo. En este caso, se dice que para las criaturas no conceptuales su relación con el mundo es de orden causal, no conceptual, por lo que el contenido debe describirse en términos causales. Si esto es así, resulta que la descripción que hemos hecho no es tendenciosa por capricho nuestro, sino por exigencias mismas del concepto de causalidad del monismo anómalo. De nuevo, el argumento es que un suceso es causal cuando tiene una descripción verdadera que lo hace una instancia de una ley de la naturaleza. Esto quiere decir que la especificación del contenido debe darse de un modo que pueda fijar condiciones de verdad, y esta exigencia fuerza la descripción del contenido en términos proposicionales, de nombres y predicados. 
El argumento general muestra que hay una preconcepción de la naturaleza que conlleva estas descripciones de los contenidos de los agentes causales, y esa preconcepción de la naturaleza está dada por la normatividad de la verdad, es decir, por la normatividad de los conceptos. Este hallazgo deja ver que las triangulaciones, como intento de mostrar las raíces naturales de la racionalidad, nos ofrecen una visión inconsistente de la naturaleza. Nos dice que es causal, extensional y no conceptual. Sin embargo, aunque en efecto tenemos que afirmar que es no conceptual, eso implica el abandono de la idea de que la naturaleza es extensional y causal, pues una naturaleza de este tipo solo es concebible por criaturas racionales. Así pues, nos encontramos en un dilema. O aceptamos que las criaturas preconceptuales son en realidad conceptuales, de modo que podemos aceptar que en efecto interactúan en el mismo mundo natural causal de hechos, propiedades y objetos, o aceptamos que las criaturas son en realidad no conceptuales y, entonces, abandonamos la idea de que hay único mundo natural causal en la triangulación transicional.

\section{Conclusión}

El principal problema metafísico de las triangulaciones y del monismo anómalo como explicación de las raíces naturales de la racionalidad es presuponer un mundo hecho ya a medida de la racionalidad como antepasado metafísico de la racionalidad misma. Buscando dar con las raíces naturales de la racionalidad, las triangulaciones solo han mostrado las fantasías de la razón que busca sus antepasados no racionales. Paradójicamente, esta circunstancia debería favorecer la empresa naturalista genética, al menos en la medida en que habría una realidad ya fija que gradualmente se iría representando de un modo cada vez más sofisticado hasta conseguir el refinamiento descriptivo que llamamos clasificación o conceptualización. Sin embargo, la conclusión es exactamente la opuesta.

La descripción ofrecida del mundo supone una metafísica asimétrica. La asimetría consiste en que no somos imparciales con respecto a la naturaleza y a la racionalidad, pues definimos la naturaleza desde la racionalidad (Cussins, 1992, p. 652). Aunque tratamos de definir el mundo y la mente en condiciones iguales, en realidad catalogamos como mundo eso que son las exigencias de cierta racionalidad. Por eso Davidson puede construir su monismo. En dicha catalogación 
nos valemos ya de los recursos que la razón misma ofrece, como la prueba de sustitución. Pero, como hemos mostrado, dicha prueba define recíprocamente la extensión y la intensión, y las caracteriza, por la definición misma, como compartimentos separados y excluyentes. Esto motiva el acertado escepticismo de los críticos de Davidson.

En este sentido, la metafísica asimétrica es la responsable de que la pregunta genética resulte tan difícil de plantear: ¿cómo se deriva la intensión de la extensión?, ¿cómo la causalidad engendra normatividad?, ¿cómo se deriva la razón de la naturaleza? En nuestra opinión, la dificultad de responder a estas cuestiones radica en que en ellas se asume que la extensión es previa a la intensión, que la causalidad es previa a la normatividad, y que esa concepción extensional y causal de la naturaleza precede a la razón, olvidando que son términos definidos recíprocamente y de modo excluyente.

Contra esta concepción de la empresa naturalista genética, en este trabajo hemos intentado mostrar que las dicotomías mencionadas se constituyen recíprocamente cuando las criaturas llegan a ser conceptuales, y que no hay entonces antepasados naturales de la razón, no al menos en esta concepción de la naturaleza. En consecuencia, un proyecto naturalista genético plausible debe modificar el contenido que le dé al monismo. No puede ser la naturaleza causal y extensional la que funda la razón. Para esbozar cuál sería dicha naturaleza es necesario corregir la asimetría metafísica diagnosticada. A lo mejor, la perspectiva genética será plausible entonces desde una metafísica simétrica.

\section{Referencias}

Carnap, R. (1956). Meaning and Necessity. Chicago: The University of Chicago Press.

Cussins, A. (1990). The Connectionist Construction of Concepts. M. Boden (Comp.), The Philosophy of Artificial Intelligence (pp. 368-440). Oxford: Oxford University Press.

Cussins, A. (1992.). Content, Embodiment and Objectivity: The Theory of Cognitive Trails. Mind, New Series, 101(404), pp. 651-688.

Cussins, A. (2002). Postscript: Experience, Thought and Activity. Y. Günther (Ed.), Essays on Nonconceptual Content (pp. 147-163). London: MIT Press. 
Davidson, D. (1995 [1963]). Acciones, razones y causas. Ensayos sobre acciones y sucesos (pp. 17-36). (Trad. O. Hansberg). México, D.F.: UNAM-Crítica.

Davidson, D. (1995 [1970]). Sucesos mentales", Ensayos sobre acciones y sucesos (pp. 263-287). (Trad. O. Hansberg). México, D.F.: UNAM-Crítica.

Davidson, D. (2001 [1975]). Pensamiento y habla. De la verdady de la interpretación (pp. 164-178). (Trad. G. Filipi). Barcelona: Gedisa.

Davidson, D. (2003 [1982]). Animales racionales. Subjetivo, intersubjetivo, objetivo (pp. 141-155). (Trad. O. Fernández). Madrid: Cátedra.

Davidson, D. (1992 [1989]). Las condiciones del pensamiento. Mente, mundo y acción (pp. 152-161). (Trad. C. Moya). Barcelona: Paidós.

Davidson, D. (2003 [1991]). Tres variedades de conocimiento. Subjetivo, intersubjetivo, objetivo (pp. 280-300). (Trad. O. Fernández). Madrid: Cátedra.

Davidson, D. (2003 [1992]). La segunda persona. Subjetivo, intersubjetivo, objetivo (pp. 156-175). (Trad. O. Fernández). Madrid: Cátedra.

Davidson, D. (2003 [1997]). La aparición del pensamiento. Subjetivo, intersubjetivo, objetivo (pp. 176-190). (Trad. O. Fernández). Madrid: Cátedra.

Davidson, D. (2001). Comments on Karlovy Vary Papers. P. Kotatko et al. (Eds.), Interpreting Davidson (pp. 285-307). Stanford: CSLI.

Eilan, N. (2005). Joint Attention Communication and Mind. N. Eilan et al. (Eds.), Joint Attention: Communication and Other Minds (pp. 1-33). Oxford: Oxford University Press.

Evans, G. (1982). The Varieties of Reference. Oxford: Oxford University Press.

Evnine, S. (1991). Donald Davidson. Oxford: Polity Press.

Fodor, J. (1994). Psicosemántica (Trad. O. González). Madrid: Tecnos.

Føllesdal, D. (1999). Triangulation. L. Hahn (Ed.), The Philosophy of Donald Davidson (pp. 719-728). Chicago: Open Court.

Glock, H.J. (2003). Quine and Davidson on Language Thought and Reality. Cambridge: Cambridge University Press.

Glüer, K. (2006). Triangulation. E. Lepore et al. (Eds.), The Oxford Handbook of Philosophy of Language (pp. 1006-1019). Oxford: Clarendon Press.

Günther, Y. (2003). General Introduction. Y. Günther (Ed.), Essays on Nonconceptual Content (pp. 1-19). Londres: MIT Press. 
Heal, J. (1997). Radical Interpretation. J. Hale et al. (Eds.), A Companion to the Philosophy of Language (pp. 175-196). Oxford: Blackwell.

Hornsby, J. (1985). Physicalism, Events and Part-Whole Relations. E. LePore et al. (Eds.), Actions and Events (pp. 444-458). Oxford: Blackwell.

Kim, J. (1998). Philosophy of Mind. Boulder: Westview Press.

Lepore, E. \& Ludwig, K. (2005). Donald Davidson. Meaning, Truth, Language and Reality. Oxford: Clarendon Press.

Macdonald, C. (1989). Mind-Body Identity Theories. London: Routledge.

Malpas, J. (1992). Donald Davidson and the Mirror of Meaning. Cambridge: Cambridge University Press.

McDowell, J. (1985). Functionalism and Anomalous Monism. E. LePore et al. (Eds.), Actions and Events (pp. 187-398). Oxford: Blackwell.

Miguens, S. (2006). Conceito de crença, triangulações e atenção conjunta. Perspectives on Rationality (pp. 99-117). Porto: Faculdade de Letras da Universidade do Porto.

Moya, C. (1990). The Philosophy of Action. Oxford: Polity Press.

Moya, C. (1992). Mente, mundo y acción. Mente mundo y acción (pp. 9-45). Barcelona: Paidós.

Pagin, P. (2001). Semantic Triangulation. P. Kotatko Jet al. (Eds.), Interpreting Davidson (pp. 199-212). Stanford: CSLI.

Pérez Jiménez, M.A. (2008). First, Second and Third Person in the Emergence of Thought. J. Padilla-Galvez (Ed.), Phenomenology as Grammar (pp. 185197). Ontos Verlag: Frankfurt am Main.

Pinedo, M. (2004). De la interpretación radical a la fusión de horizontes. J.J. Acero et al. (Eds.), El legado de Gadamer (pp. 225-235). Granada: Universidad de Granada.

Quine, W.v.O. (1998). Del estímulo a la ciencia (Trad. J. Pagés). Barcelona: Ariel. Ramberg, B. (1989). Donald Davidson's Philosophy of Language. Oxford: Blackwell. Tye, M. (2006). The Thesis of Nonconceptual Content. Ch. Van Geen et al. (Eds.), The Structure of Nonconceptual Content, European Review of Philosophy, 6 (pp. 7-30). Stanford: CSLI. 